\title{
Vehicle Tracking System using GPRS
}

\author{
S. Ikram $^{1} \&$ F. T. Shah ${ }^{2}$ \\ ${ }^{1}$ Seidco Communication L.L.C, Abu Dhabi, UAE \\ ${ }^{2}$ COMSATS Institute of Information Technology, Lahore, Pakistan
}

\begin{abstract}
The demands on Vehicle Tracking Systems are increasing day by day, as increasing car theft becomes one of the most challenging problems faced by our modern society, especially for security agencies, ambulances, police and even personal usage. We propose a client/server solution for transferring the required information which has an automatic updating facility. We have introduced a new Technology named "General Packet Radio Services (GPRS)" in this application. We are transferring data using GPRS Services to and from the Base Station and the Remote Station.

Keywords: GSM, SMS, GPRS, GIS, GPS, digitization, positioning, blocking.
\end{abstract}

\section{Introduction}

The Vehicle Tracking System allows a user to monitor the positions of multiple vehicles and disable them remotely in case of theft. In this project our first completed task was the Map Digitization (GIS and GPS Solution). GIS and GPS technology were linked with means of wireless communication, essential to the system determining the vehicle location [1]. It is necessary to have a technique to handle the huge amount of spatial data entailed in a digital road map in order to trace the accurate position within a reasonable time. Our main focus was on the practical design (device Integration) and implementation.

For the digitization process, we felt that the development of a new software tool was required. Although there is a big market for related software from Geographical Information Systems (GIS), just to gain experience we started on our own.

In many fields of research, valuable information is stored in atlases and maps which are only available as printed media. Scientists who are interested in 
computational analysis often seek to digitize the data to make it accessible for numerical calculations.

In this application, we used a hand-held GPS receiver to perform georeferencing, plotting real-time points (longitude and latitude) from actual locations on a map. These points were saved as boundary coordinates for the map using VB/VB.Net software. The map was then scanned and digitized, and the boundary coordinates used to give the angle for proportion (tilting) and therefore, and actual north-east view. Mathematical functions were then used to spread this angle information over the whole map. A software tool has been built in such as way that the application is capable of showing the exact location of the vehicle according to GPS (longitude and latitude) values. The user can scroll through this map, make restricted areas [zones], zoom-in /zoom-out, pan, and so on.

Previous tracking systems were based on SMS (based on Circuit Switching Mode) communication, but we used GPRS (based on Packet Switching Mode) for tracking the vehicles. This way, data appears as live tracking (updating every second). Another main advantage of GPRS is the cost. We can transfer data in a unit of KB/ MB (very cheap), as compared to SMS (max. 160 characters).

General Packet Radio Services (GPRS) is a wireless communication solution built on the existing GSM network. It provides much needed packet data services to most areas of the world. We have implemented a GPRS solution for a vehicle tracking system to enable data transfer using packet switched mode.

Using GPRS, we can transfer large amount of data in a very short time and in a very cheap price for a large amounts of data, for example, it will allow us to send or receive large files (Mega Bytes) within seconds. GSM is based on circuit switched mode that transfers data character by character and has many limitations, for example, a maximum of 160 characters per SMS, and charges based on the duration of the call.

GPRS allows us to use the Internet facility in a very speedy way (maximum of $171.2 \mathrm{kbps}$ ) using the packet data services and WAP (Wireless Application Protocol) Technology on our cell phone. Using WAP, we can transfer compressed data files through TCP/IP. For the practical implementation of GPRS for data transfer, we are using WinSock (Windows Socket) to enable both the server and client to communicate using the same protocol.

\section{Project vision}

This system is designed in SEIDCO Communication to aid with stolen car recovery. Car theft is a major concern in our society, especially in Asian and African regions, and as every year car prices rise, cars are becoming very valuable assets. Buying a car is a major investment for many people and protecting that investment is important.

To solve this problem, this VTS system can stop the theft vehicle upon the owner's request at any time, warning the driver first by generating alarms or voice messages. Another major concern is the inappropriate or unauthorized 
usage of company vehicles by employees. Information can be provided regarding the drivers attitude, speeding, harsh braking, etc. and give the location of the vehicle at all times [6].

Another application could be to provide information regarding the circumstances immediately preceding an accident. For example, was the seat belt fastened, speed of the vehicle, etc [6]. That is why this system will give car owners the piece of mind they deserve.

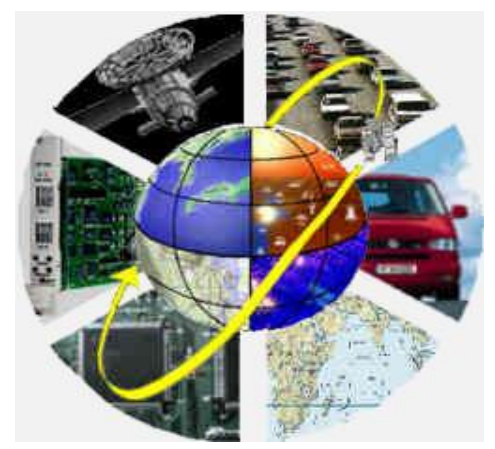

Figure 1: Global vision.

\subsection{Functionalities}

- $\quad$ Locate the car position (longitude and latitude).

- Display the position on a digitized map.

- Disable the car remotely on the owner's request [2].

- Provide information regarding speed limitations, seat belt, harsh breaking, etc., to be able to record any rule violations.

- Generate reports, for example, driver performance, usage of vehicle, violation of driving rules, etc.

\subsection{Additional features}

- We can have live tracking using GPRS and this is what we need to implement for our vehicles. It can send the position of vehicles every second (depending upon the refresh rate of the GPS receiver and GPRS data transfer speed [2]) to the base station and the user can view as live tracking.

- We can watch live footage of the traveled path of the vehicle if we attach a digital camera to the vehicle.

\subsection{External interface}

- Vehicle control device (Black Box).

- $\quad$ Embedded GPS module with external antenna.

- $\quad$ Embedded GSM / GPRS module with external antenna. 
- GPRS/GSM modems with antenna (base station).

- Network connection (mobile SIM card).

- Disabling circuits or alarms with sensors (optional).

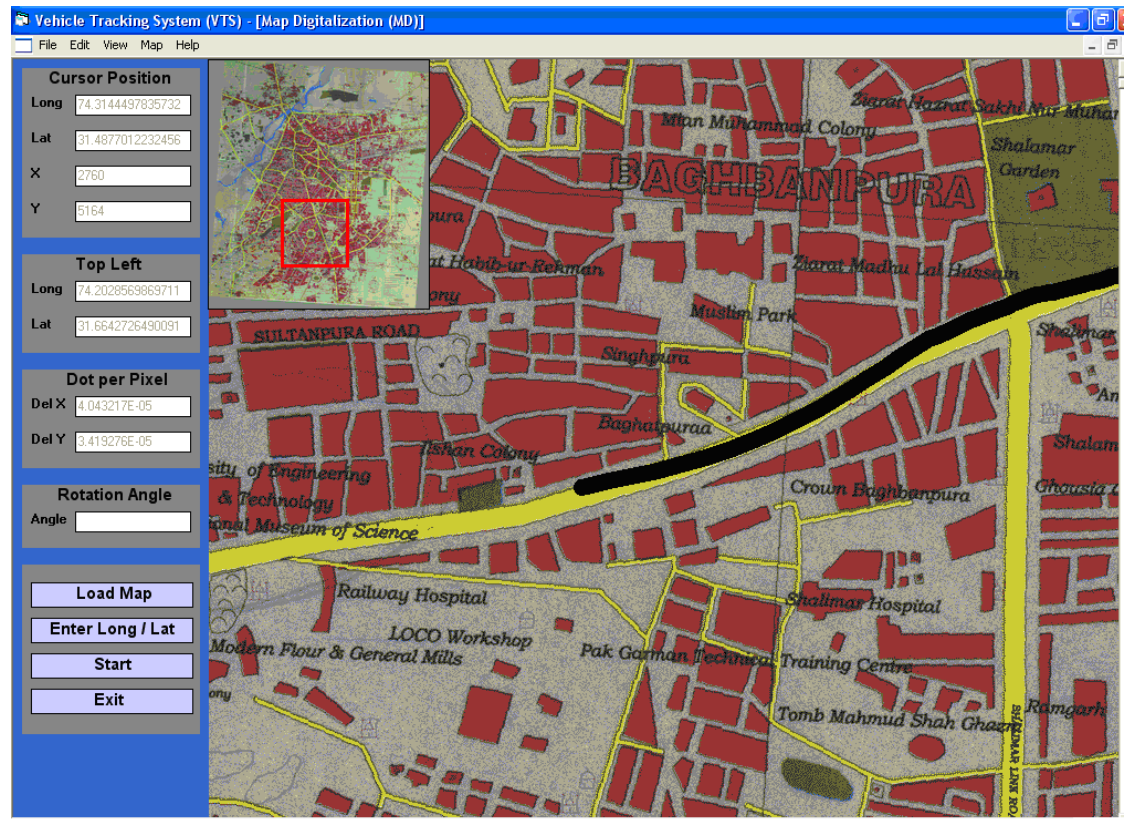

Figure 2: $\quad$ Software application view.

\subsection{Functional requirements}

The functional requirements consist of GPS and GPRS modules, control unit, desktop computer with VTS application, and digitized maps for the specific area according to user requirement. GSM/GPRS modems and a user-friendly software interface.

\subsection{Performance requirements}

To ensure the hardware system offers the specified functions, it has to execute according to the following specifications:

- The signal position data must be sent every second to track the vehicular position [4].

- Transfer of the inputs and outputs for the System will be made via modems.

- The signal contains information that identifies the vehicle and the action being performed. 
- The software group will determine the codes for the action and the vehicular identification system.

- The signal sent from the car must be translated into location information that can be mapped on the desktop computer.

- The system can disable the vehicle in case of theft or any other serious problem.

\section{Why GPRS}

- Sharing frequency channel.

- Stay connected, charged only on data transfer.

- Maximum practical transfer rate is $115 \mathrm{kbps}$.

- $\quad$ Priority-based architecture.

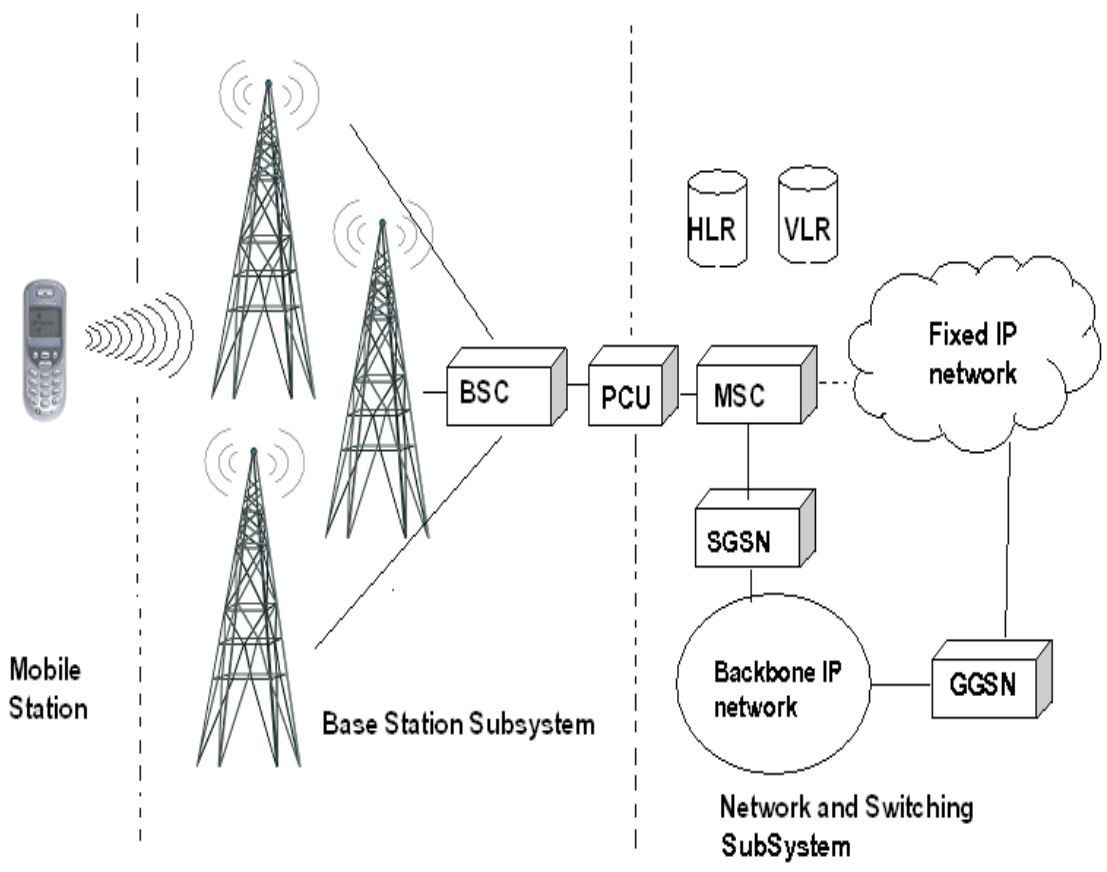

Figure 3: $\quad$ GPRS network view.

\section{Business strategy}

In UAE, there are many companies offering a Vehicle Tracking System with a GSM (SMS) and GPS facility to get the current position of the vehicle, and GSM Data Call Technology to download the saved longitudes and latitudes of the traveled path. However, it is much more expensive compared to GPRS because of the deduction of charges and the data limitation against each SMS and per 
minute limitations against the data call depending on the duration [3]. In addition, the major issue is that both these technologies can only transfer text data, not data such as image files, voice files, etc.

In GPRS, we can transfer data using GPRS enabled SIM and it will allow us to transfer almost all kind of files, it will not depend on the file format but just the packet size for transfer. Therefore, if we compare it with any other technology for tracking purposes, we will see that GPRS is almost 10 times cheaper and faster than SMS or Data Call.

For this VTS application in UAE, we applied both technologies as optional features. Due to the non-availability of GPRS in certain (desert) areas, the system will automatically switch to GSM (SMS) mode.

\subsection{Criterion to compare project solution merit}

\subsubsection{Cost}

Cost is the primary design consideration that will be used to determine hardware and software. Besides project budget considerations, this system will need to have a unit cost as low as possible for mass distribution [2].

\subsubsection{Size}

The system will need to be able to be install in a vehicle, such that the system will not interfere with operation and will be hard to notice or disable. Size is a primary consideration to determine available mounting options [2].

\subsubsection{Durability}

Clarity of signal and low maintenance will be essential in guaranteeing that the system will operate when needed.

\subsubsection{Skills}

The implementation of this system should be invisible to the automotive operator and simple to use by the operator. The amount of training required to use the system effectively will need to be factored into the design method to facilitate ease of use.

\subsection{Procedure of transferring data}

- $\quad$ First of all both communication ends should have GPRS enabled SIM with GPRS modems, also should be in always connected mode.

- One end should be treated as a server with the other as a client. The client must have the IP address of the server because of the remote connection for file transfer using TCP/IP protocol and WinSock (Windows Socket), for windows applications.

- The client pings the server through the given IP address to establish the direct communication session, if the IP is correct then the connection will be established and vise versa. This connection will be treated as a GPRS connection. 
- Now the client can send any kind of file to the server and this filetransferring mode will be known as Packet Switching Data Mode.

- To get the current location of a vehicle, the server will also follow the same procedure, as to ping the client on a particular IP and create a session. The client will send back the position against the request of server.

\subsection{Using packet switched data}

\subsubsection{Advantages}

- Better allocation of resources because different users can share the same frequency channel when online.

- User can stay connected to the network, since user will be charged based on data transfer, not the duration of connection.

- Package data is smaller than circuit-switched data; therefore, packages can fit easily between other user's data packages.

- Due to multiple time-slot architecture, transfer rates can reach a maximum of $115 \mathrm{kbps}$ practically [5].

- Packet-switched is more cost-efficient because the same frequency channel can support more mobile users. This means it has increased the capacity of the network [5].

- Since packages can easily fit into frequency networks that means mobile users can shorten the connection time when initiating a data transfer.

- Priority-based architecture - makes sure that important data can have access to the network. For example, voice data always have higher priority over message data [5].

- Using Fixed IP (Lease Line) policy for Internet connection, we can omit GSM/GPRS modem from the base station (optional), in this way it will only support GPRS, not the SMS feature.

\subsubsection{Disadvantages}

- Mobile user is not guaranteed to have an available frequency channel even if the user is connected to the GPRS network.

- Mobile user is connected to the network; however, a frequency channel is assigned to the mobile user only when data is being transferred. Therefore the mobile user may not have access to frequency channel.

- Difficult to implement package data

\section{Acknowledgements}

This Paper was prepared in collaboration with Seidco Communication L.L.C, UAE and the Department of Computer Sciences, COMSATS Institute of Information Technology, Lahore, Pakistan.

The Paper was extensively discussed in a series of meetings and extensive exchanges over a period of one year. Those who directly involved were: $\mathrm{Mr}$ 
Nadeem Tahir (MS-CS Student, New York, USA), Mr Imran Arshad (Communication Engineer, Pakistan), Eng. Omar Ajjaoui (CAO, Seidco Communication, UAE) and Ms Nermin Gamal (System Analyst, UAE)

\section{References}

[1] Maguire, D.J., An Overview and Definition of GIS, Geographical Information Systems, 1, pp. 9-20, 1991.

[2] http://www.mobiletrackingsystems.com/

[3] French, R.L., Land Vehicle Navigation and Tracking, Global Positioning System: Theory and Applications, 164, pp. 275-301, 1996.

[4] Braff, R., Applications of the GPS to Air Traffic Control, Global Positioning System: Theory and Applications, 164, pp. 327-374, 1996.

[5] Kari, H.H. and Helsinki University of Technology, General Packet Radio Service (GPRS), 2001 http://www.ee.oulu.fi/ fiat/gprs. html

[6] Shladover, S.E., Research and Development Needs for Advanced Vehicle Control Systems, IEEE Computer Society, 1993 